\title{
CT Image Reconstruction Approaches Applied to Time-Frequency Representation of Signals
}

\author{
Jozef Púčik \\ Department of Radio and Electronics, Faculty of Electrical Engineering and Information Technology, \\ Slovak University of Technology in Bratislava, Ilkovičova 3, 81219 Bratislava, Slovak Republic \\ Email:pucik@elf.stuba.sk \\ Rami Oweis \\ Biomedical Engineering Department, Faculty of Engineering, Jordan University of Science and Technology, \\ Irbid 22110, Jordan \\ Email: oweis@just.edu.jo
}

Received 29 January 2002 and in revised form 22 October 2002

\begin{abstract}
The mathematical formulation used in tomography has been successfully applied to time-frequency analysis, which represents an important "imaging modality" of the structure of signals. Based on the interrelation between CT and time-frequency analysis, new methods have been developed for the latter. In this paper, an original method for constructing the time-frequency representation of signals from the squared magnitudes of their fractional Fourier transforms is presented. The method uses $\alpha$-norm minimization with $\alpha \rightarrow 1$ which is motivated by Rényi entropy maximization. An iterative optimization method with adaptive estimation of the convergence parameter is elaborated. The proposed method exhibits advantages in the suppression of interference terms for signals with simple time-frequency configurations.
\end{abstract}

Keywords and phrases: Radon-Wigner transform, Wigner distribution, fractional Fourier transform, Rényi entropy.

\section{INTRODUCTION}

Time-frequency representation (TFR) [1] is an important tool for nonstationary signals and time-varying systems analysis or synthesis. Constructing such a representation, clearly and accurately describing the energy distribution of the signal over the time-frequency plane, is therefore of fundamental importance.

Prior to presenting the mathematical background of the proposed method, the concept of the time-frequency plane and the constrains imposed on the TFR in order to yield a reasonably accurate time-frequency description will be briefly reviewed. Continuous-time complex-valued deterministic signals with finite energy will be considered.

In the time domain, a signal is completely described by a function $s(t)$ and its instantaneous power by $|s(t)|^{2}$. A time-varying instantaneous frequency can be associated with the signal at each time $t$. By analogy, in the frequency domain, the signal may be described by its Fourier transform (FT), and its energy distribution over frequency given by the Fourier transform squared. A concept dual to the instantaneous frequency is the group delay, which characterizes the occurrence of a frequency component in time.

The time-frequency representation $\operatorname{TFR}_{s}(t, \omega)$ charac- terizes the energy distribution of a signal over the timefrequency plane. As an energy distribution, this function should be nonnegative everywhere, analogous to the simultaneous probability density function of a 2-dimensional random vector. The energy spectrum and the instantaneous power (which represent the marginal densities of the joint time-frequency distribution) should be obtained by integrating the distribution along the time and frequency axes, respectively. This property, referred to as the time-frequency marginals, strongly influences the time-frequency resolution. These and many other properties (e.g., the first-order conditional moments which represent the instantaneous frequency and group delay) are generally accepted as desirable properties of the TFR [2]. Unfortunately, no distribution can satisfy all these properties simultaneously.

Although a notion of "ideal" time-frequency distribution has been mentioned in some works, the situation in timefrequency analysis is controversial and ideal time-frequency distribution cannot exist. Nowadays, various methods for time-frequency analysis are frequently published. Because infinite resolution cannot be achieved in both time and frequency simultaneously, various compromises have been proposed. The most commonly used techniques are Cohen class distributions which are briefly discussed in Section 2. Instead 
of directly transforming a time-domain function by integration to yield a time-frequency representation, a general

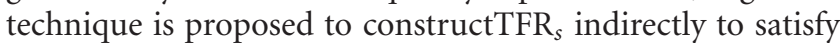
certain predetermined criteria such as maximum entropy. This paper applies this technique to the case of generalized marginals (Section 3).

The main part of this paper (Section 4) is devoted to numerical methods for reconstruction of a TFR from an incomplete set of fractional Fourier transforms (FrFT) by means of optimization techniques. Since Shannon entropy maximization method cannot be used generally due to the nonnegativity requirement, Rényi entropy is chosen as an alternative reconstruction functional. A gradient-based method (derived by the technique of Lagrange multipliers) with adaptive estimation of a convergence parameter is developed and applied to various signals (Section 5).

\section{COHEN CLASS}

The Wigner (Wigner-Ville) distribution (WD) $[1,3]$ is a principal representative of distributions which describe the time-frequency energy distribution; the WD is defined as the FT of the instantaneous autocorrelation function

$$
r_{s}(t, \tau)=s\left(t+\frac{\tau}{2}\right) s^{*}\left(t-\frac{\tau}{2}\right)
$$

(where $*$ denotes complex conjugation), that is,

$$
\mathrm{WD}_{s}(t, \omega)=\int r_{s}(t, \tau) \mathrm{e}^{-j \omega \tau} d \tau
$$

The WD is a real-valued function, often assuming negative values. WD-based analysis results in an intuitive interpretation only for certain types of signals (namely, linear chirps and Dirac pulses in either the time or frequency domain). The analyzed signal is usually considered to be a composition of signals with simple TFR, called components. In general, due to the nonlinearity of the $\mathrm{WD}$, interference terms (cross-terms) may result, such as intercomponent interference and internal interference in the case of nonlinear frequency modulation [1]. These undesirable terms can have amplitudes exceeding those of actual autocomponents. (Interference structure of WD reflects also phase shift between components that can be of interest in some application, but phase relations cannot be simply identified in WD image.) Due to these phenomena, a smoothing filter is applied to the result of the WD in order to suppress interference and noise.

The WD (possibly followed by linear, time-invariant filtering) has two noteworthy features: the order of nonlinearity is quadratic and the resulting distributions are timefrequency shift covariants. Such distributions have been unified by Cohen. The general form of the shift-covariant distributions is given by [4]

$$
C_{s}^{(\phi)}(t, \omega)=\frac{1}{2 \pi} \iiint \phi(\theta, \tau) r_{s}\left(t^{\prime}, \tau\right) \mathrm{e}^{-j \omega \tau-j \theta t+j \theta t^{\prime}} d \theta d \tau d t^{\prime},
$$

where $\phi$ denotes the distribution kernel.

\section{RADON-WIGNER TRANSFORM}

\subsection{Time-frequency marginals}

The instantaneous power and the energy spectrum are considered as desired densities over time and frequency, respectively. The representation $\operatorname{TFR}_{s}(t, \omega)$ satisfies the abovementioned marginal property if the univariate densities determined by the integration of a joint density function along $t$ and $\omega$ variables fulfill the following relations:

$$
\begin{aligned}
\frac{1}{2 \pi} \int \operatorname{TFR}_{s}(t, \omega) d \omega & =|s(t)|^{2}, \\
\frac{1}{2 \pi} \int \operatorname{TFR}_{s}(t, \omega) d t & =|S(\omega)|^{2},
\end{aligned}
$$

where $S(\omega)$ denotes the unitary version of the Fourier transform

$$
S(\omega)=\frac{1}{\sqrt{2 \pi}} \int s(t) \mathrm{e}^{-j \omega t} d t .
$$

Cohen and Posch [5] have shown that nonnegativevalued distributions with correct marginals exist; minimizing cross-entropy is an attractive method for constructing nonnegative distributions [6]. This method yields a solution which may be written as a product of three functions, namely, prior distribution function, time-dependent function, and frequency-dependent function. In practical applications, this separability simplifies the mathematical derivation and decreases the computational complexity, but unfortunately the solution obtained is not sufficiently general to express complex TFR. This drawback arises mainly in the case of uniform prior distribution and can be compensated by imposing additional constraints based on FrFT and line integrals along paths not parallel to either the time or frequency axes [7].

\subsection{Fractional Fourier transform (FrFT)}

The conventional marginals (4) may be viewed as specific cases $(\varphi=0$ or $\pi / 2)$ of the generalized marginals, expressed as [8]

$$
\frac{1}{2 \pi} \mathcal{R}_{\varphi}\left\{\operatorname{TFR}_{s}(t, \omega)\right\}(u)=\left|S_{\varphi}(u)\right|^{2},
$$

where $\mathcal{R}_{\varphi}$ denotes the Radon transform operator, $\varphi$ is the rotation angle of the time-frequency plane, and $S_{\varphi}(u)$ is the FrFT of $s(t)$. The existence of such a TFR satisfying (6) is obvious as a consequence of the well-known Fourier-slice theorem. The geometrical interpretation of the rotation angle $\varphi$ depends on the used time and frequency unit. To avoid this ambiguity, a time-scaled signal $s_{a}(x)=a^{1 / 2} s(a x)$, as a function of the dimensionless time-variable $x$, may be used instead of $s(t)$. The FT of $s_{a}(x)$ (with respect to the variable $x)$ is $S_{a}(y)=a^{-1 / 2} S\left(a^{-1} y\right)$, where $y$ is the dimensionless angular frequency. The common choice for the scaling factor $a$ is $a=\left(t_{m} / \omega_{m}\right)^{1 / 2}$ provided that intervals $\left(-t_{m}, t_{m}\right)$ and $\left(-\omega_{m}, \omega_{m}\right)$ represent the time and frequency supports of the signal [9]. This particular option reshapes the rectangular time-frequency support into a square-shaped region. 
The FrFT is a unitary transformation defined as [10]

$$
S_{\varphi}(u)=\mathcal{F}_{\varphi}\{s(t)\}(u)=\int s(t) B_{\varphi}(t, u) d t,
$$

where $B_{\varphi}(t, u)$ is the following transform kernel:

$$
\begin{aligned}
& B_{\varphi}(t, u) \\
& = \begin{cases}\delta(t-u), & \varphi=0, \\
\delta(t+u), & \varphi= \pm \pi, \\
\frac{e^{j[\varphi / 2-(\pi / 4) \operatorname{sgn}(\varphi)]}}{\sqrt{2 \pi|\sin \varphi|}} e^{j\left(\left(u^{2}+t^{2}\right) / 2\right) \cot \varphi-j u t \csc \varphi}, & \varphi \in(-\pi, 0) \cup(0, \pi), \\
B_{\varphi \pm 2 n \pi}(t, u), & |\varphi|>\pi, n \in \mathbb{Z} .\end{cases}
\end{aligned}
$$

Note that the FrFT incorporates the identity $\left(S_{0}(u)=s(u)\right)$ and the ordinary FT $(5)\left(S_{\pi / 2}(u)=S(u)\right)$ as specific cases of (7) for $\varphi=0$ and $\varphi=\pi / 2$, respectively. The FrFT effectively results in a rotation of the time-frequency plane; it is easy to show that the inverse transform kernel $B_{\varphi}^{*}(t, u)$ is the eigenfunction of the time-frequency operator

$$
\hat{u}=\hat{t} \cos \varphi+\hat{\omega} \sin \varphi,
$$

where $\hat{\omega}=(1 / j)(\partial / \partial t)$ and $\hat{t}=t$, likewise, the inverse FT kernel $\mathrm{e}^{j \omega t}$ is the eigenfunction of the frequency operator $\hat{\omega}$. Mean values (first- and second-order moments) of the operator $\hat{u}$ characterize the average location and the spread of the signal in the time-frequency plane with respect to the lines $t \cos \varphi+\omega \sin \varphi=$ const. In general, the inverse of the FrFT is a decomposition of a signal using an orthonormal basis of linearly frequency-modulated functions. The linear frequency modulation may be recognized in FrFT kernel as a quadratic-phase term; other terms ensure unitarity and angle additivity properties of the transform [8].

\subsection{Reconstruction of WD}

The WD as specific case of TFR satisfies generalised marginals (6) for all angles $\varphi \in\langle 0, \pi)$ [8]. Magnitude-squared FrFT is then referred to as the Radon-Wigner transform of signal $s(t)$. As derived originally by J. Radon, a function of two variables (such as an image) may be uniquely reconstructed from a full set of its line integrals. Specifically, the WD may be computed from the squared magnitude of the FrFT $m_{\mathrm{WD}_{s}}(u, \varphi)=2 \pi\left|S_{\varphi}(u)\right|^{2}$ of the signal by the following inversion formula:

$$
\begin{aligned}
\mathrm{WD}_{s}(t, \omega)=\frac{1}{4 \pi^{2}} \int_{0}^{\pi} \int_{-\infty}^{-\infty} \int_{-\infty}^{\infty} & m_{\mathrm{WD}_{s}}(u, \varphi) \mathrm{e}^{-j u \rho} \\
& \times \mathrm{e}^{j \rho(t \cos \varphi+\omega \sin \varphi)}|\rho| d u d \rho d \varphi .
\end{aligned}
$$

Computation of (10) is usually divided into several steps, the arrangement of which results in various techniques for the TFR construction. One such technique, that is commonly used, is the filtered back-projection method. In practice, the filtering, which is equivalent to differentiation followed by
Hilbert transformation, usually involves additional smoothing and spectral limiting. The additional filter transfer function $G(\rho, \varphi)$ can be interpreted as the Cohen class kernel $\phi(\theta, \tau)$ expressed in polar coordinates. This approach is unsuitable in terms of computational efficiency compared to conventional procedures for computation of the Cohen distribution, which use Cartesian time-frequency coordinates. However, using polar coordinate kernels simplifies the formulation of generalized marginal consistency conditions. Conditions for other properties can also be reformulated for the filter transfer function $G(\rho, \varphi)$ instead of the $\phi(\theta, \tau)$ kernel.

\section{ITERATIVE RECONSTRUCTION OF THE TFR}

As discussed in Section 3.3, filtered back-projection approach in the time-frequency representation results in Cohen class distributions. Since these distributions offer a trade-off between the cross-terms suppression and the autocomponent resolution, we will consider an optimization-based inversion from small number of projections as an alternative to the direct linear methods. The constraint imposed is that the generalized marginals (6) are correct for several angles $\varphi_{1}, \ldots, \varphi_{M}$, that is,

$$
\mathcal{R}_{i}\{T(t, \omega)\}(u)=m_{i}(u), \quad i=1, \ldots, M,
$$

where $T$ is to be reconstructed and $m_{i}(u)=2 \pi\left|S_{\varphi_{i}}(u)\right|^{2}$.

The constraint of correct marginals when combined with optimization procedures (e.g., least squares method, entropy maximization, and cross-entropy minimization) provides a tool for time-frequency distribution construction $[6,7,11$, 12]. In the case of Shannon entropy maximization with generalized marginals as constraints, Lagrange multipliers technique with nonlinear Gauss-Seidel-type iteration procedure may be advantageously used [13]. In this particular method, Lagrange multipliers are repeatedly and successively updated for $i=1, \ldots, M$ to satisfy (11); only one marginal is assumed to be exactly correct in each iteration. The iteration procedure converges provided that a nonnegative solution exists.

Unfortunately, Shannon-entropy-based methods may fail because the nonnegative TFR with correct marginals may not exist. For this reason, the application of an adapted Rényi entropy seems to be more suitable for TFR reconstruction.

\subsection{Rényi entropy of TFR}

The Rényi entropy [14] is a generalization of the Shannon entropy, which is defined for the discrete probability function $\mathbf{p}=\left\{p_{1}, \ldots, p_{N}\right\}$ as

$$
H(\mathbf{p})=-\sum_{i=1}^{N} p_{i} \log _{2} p_{i}
$$

Roughly spoken, Shannon entropy is a weighted arithmetic mean of the individual components $-\log _{2} p_{i}$ with weights $p_{i}$. 
This arithmetic mean may be further generalized by defining a continuous monotonic function $f(x)$ to give

$$
f^{-1}\left(\sum_{i=1}^{N} p_{i} f\left(-\log _{2} p_{i}\right)\right)
$$

Choosing $f(x)=2^{(1-\alpha) x}$, where $\alpha$ represents the order of Rényi entropy (instead of $f(x)=a x+b$ in the case of an arithmetic mean), corresponds to the Rényi entropy of order $\alpha$,

$$
H_{R}^{(\alpha)}(\mathbf{p})=\frac{1}{1-\alpha} \log _{2}\left(\sum_{i=1}^{N} p_{i}^{\alpha}\right)
$$

Rényi entropy retains most of the basic properties of Shannon entropy $[14,15]$. Moreover, as $\alpha$ approaches 1, Rényi entropy reduces to Shannon entropy. Since in many applications Shannon entropy maximization yields excellent results, this in turn will be of great help in determining the proper order $\alpha$.

Extending (14) to bivariate continuous functions yields the Rényi entropy of the TFR, which has the form

$$
H_{R}^{(\alpha)}\left(\mathrm{TFR}_{s}\right)=\frac{1}{1-\alpha} \log _{2}\left(\iint\left(\frac{\mathrm{TFR}_{s}(t, \omega)}{2 \pi E_{s}}\right)^{\alpha} d t d \omega\right),
$$

where the signal energy $E_{s}$, given by

$$
E_{s}=\frac{1}{2 \pi} \iint \operatorname{TFR}_{s}(t, \omega) d t d \omega,
$$

normalizes the $\mathrm{TFR}_{s}$.

Regardless of the probability distribution interpretation of TFR, negative values for integer orders $\alpha$ may be formally accepted in (15) provided that the integral argument also in (15) yields positive value. In the context of timefrequency analysis, $H_{R}^{(\alpha)}\left(\mathrm{TFR}_{s}\right)$ has been proposed in $[16,17]$ to measure signal complexity interpreted as a number of signal components. For this purpose, the order $\alpha$ equal to three has been preferred in [16] since the argument of the logarithm in (15) takes on nonpositive values only exceptionally, and the cross-components contributions to this term asymptotically vanish. In this paper, a maximization of Rényi entropy for a noninteger $\alpha$ close to 1 , namely, in the range $(1,2)$, is considered. Since $\alpha$ th power, as a real-valued function, does not allow negative values, absolute values will be used, consequently, the $H_{R}$ maximization is reduced to $\alpha$ norm minimization. This simple modification ensures convexity of the resulting objective functional. The convexity is a valuable property in optimization procedures, and in the case of Shannon entropy, it cannot be achieved by replacing negative values with its absolute values. It should be noted that the special case of $\alpha=2$ is equivalent to the minimum Euclidean norm solution, which can be obtained iteratively or analytically (the latter in the case of circular support [18]). An iterative procedure for $\alpha \in(1,2)$ is presented below.

\subsection{Algorithm description}

Optimization may be formulated as minimization of the following convex functional:

$$
\iint_{D}|T(t, \omega)|^{\alpha} d t d \omega
$$

over a predefined area of support $D$ subject to the generalized marginals (11) for a set of angles $\varphi_{1}, \ldots, \varphi_{M}$. The constrained optimization problem can be solved by the method of the Lagrange multipliers. The Lagrangian in this case may then be written as

$$
\begin{aligned}
L(T, & \left.\lambda_{1}, \ldots, \lambda_{M}\right) \\
= & \frac{1}{\alpha} \iint_{D}|T(t, \omega)|^{\alpha} d t d \omega \\
& \quad-\sum_{i=1}^{M} \int_{D_{i}} \lambda_{i}(u)\left[\mathcal{R}_{i}\{T(t, \omega)\}(u)-m_{i}(u)\right] d u \\
= & \frac{1}{\alpha} \iint_{D}|T(t, \omega)|^{\alpha}-\sum_{i=1}^{M} \lambda_{i}\left(t \cos \varphi_{i}+\omega \sin \varphi_{i}\right) T(t, \omega) d t d \omega \\
& +\sum_{i=1}^{M} \int_{D_{i}} \lambda_{i}(u) m_{i}(u) d u .
\end{aligned}
$$

For notational convenience, a back-projection operator $\mathcal{B}_{i}$, projecting the Lagrange multipliers back to the support of the TFR, is introduced. So,

$$
\mathcal{B}_{i}\left\{\lambda_{i}(u)\right\}(t, \omega)=\lambda_{i}\left(t \cos \varphi_{i}+\omega \sin \varphi_{i}\right) .
$$

Variation of (18) with respect to $T$ and then setting the result of variation to be zero yield an expression for TFR in terms of Lagrange multipliers

$$
\begin{aligned}
& T(t, \omega) \\
& =\left|\sum_{i=1}^{M} \mathcal{B}_{i}\left\{\lambda_{i}(u)\right\}(t, \omega)\right|^{1 /(\alpha-1)} \operatorname{sgn}\left[\sum_{i=1}^{M} \mathcal{B}_{i}\left\{\lambda_{i}(u)\right\}(t, \omega)\right] .
\end{aligned}
$$

Substituting (20) into (18) yields the dual Lagrangian

$$
\widetilde{L}\left(\lambda_{1}, \ldots, \lambda_{M}\right)=\frac{1-\alpha}{\alpha} \iint\left|\sum_{i=1}^{M} \mathcal{B}_{i} \lambda_{i}\right|^{\alpha /(\alpha-1)}+\int \sum_{i=1}^{M} p_{i} \lambda_{i},
$$

where the formal arguments of the functions of $t, \omega$, and $u$ have been omitted and integration is performed over the corresponding support regions $D$ and $D_{i}$.

This dual Lagrangian is clearly a concave functional (for $\alpha>1$ ). The solution involves finding the nonconstrained maximum of the dual Lagrangian as a functional in terms of Lagrange multipliers, for which an iterative algorithm has been developed. In each iteration, the Lagrange multipliers are updated according to the following formula, which makes use of a convergence parameter $\mu_{n}$ that must be estimated 
separately,

$$
\lambda^{(n+1)}(u)=\lambda^{(n)}(u)-\mu_{n} \mathbf{e}\left(\lambda^{(n)}(u)\right)
$$

where $\lambda^{(n+1)}(u)=\left[\lambda_{1}^{(n)}, \ldots, \lambda_{M}^{(n)}\right]^{T}$, and

$$
\mathbf{e}=\left[e_{1}, \ldots, e_{M}\right]^{T}, \quad e_{i}=\mathcal{R}_{i} T^{(n)}-m_{i},
$$

represents the error of the marginals of (11) in the $n$th iteration. The convergence parameter $\mu_{n}$ may be estimated (see the appendix) as

$$
\mu_{n} \approx \frac{2(\alpha-1)}{M}\left[\max _{i, u}\left\{\mathcal{R}_{i}\left|\sum_{j=1}^{M} \mathcal{B}_{j} \lambda_{j}^{(n)}\right|^{(2-\alpha) /(\alpha-1)}\right\}(u)\right]^{-1} .
$$

In the finite-dimensional case, the recursion (22) may be viewed as an adaptive, gradient-based (steepest-ascent) maximization of the dual Lagrangian (21).

\section{DISCUSSION}

The performance of the method has been characterized for several types of signals; results for single-component signal, multicomponent signal, and signal with nonlinear frequency modulation are presented. Discrete-time signals have been numerically synthesized; number of samples and sampling frequency determine the time and frequency supports of signals. With regard to the appropriate time scaling described in Section 3.2, optimization procedure has been performed on a square-shaped support with the well-defined rotation angles. Results of the analysis are presented graphically in Figures $1,2,3$, and 4 , where, for convenience, the time axis is labeled in samples and the frequency is normalized, with the value one corresponding to half the Nyquist frequency.

In the case of monocomponent signals, no significant differences were found compared to the WD result. As an example, a 101-sample linear frequency-modulated signal with a Gaussian amplitude envelope (the so-called "time-frequency atom") is used. The result of reconstructing a TFR for $\alpha=1.1$ and $\alpha=1.9$ for five uniformly distributed angles $\left(0^{\circ}, 36^{\circ}\right.$, $\left.72^{\circ}, 108^{\circ}, 144^{\circ}\right)$ is depicted in Figure 1. In this figure, the effect of the Rényi entropy order $\alpha$ can be seen. As $\alpha$ approaches 1, the TFR approaches the maximum Shannon entropy solution which in most cases is identical to the WD result.

Whereas in the case of monocomponent signals, noticeable differences were not expected between the proposed method and the WD result, the situation is different for multicomponent signals. The next example demonstrates that, for signals with trivial time-frequency distributions, both the suppression of interference and retention of the autocomponents are possible. In Figure 2, the analyzed signal consists of two time-frequency components with different frequencies and $\cos ^{2}$ amplitude envelopes nonoverlapping in time. In Figure 2b, the TFR was constructed using Shannon entropy method from four projections $\left(\varphi=0^{\circ}, 90^{\circ}, 45^{\circ},-45^{\circ}\right)$. In Figure $2 \mathrm{a}$, oscillatory interference appearing midway between autocomponents can be clearly observed. As known

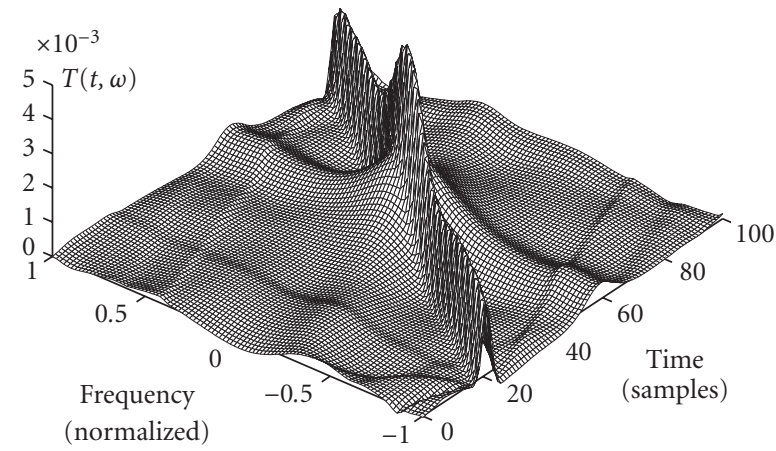

(a)

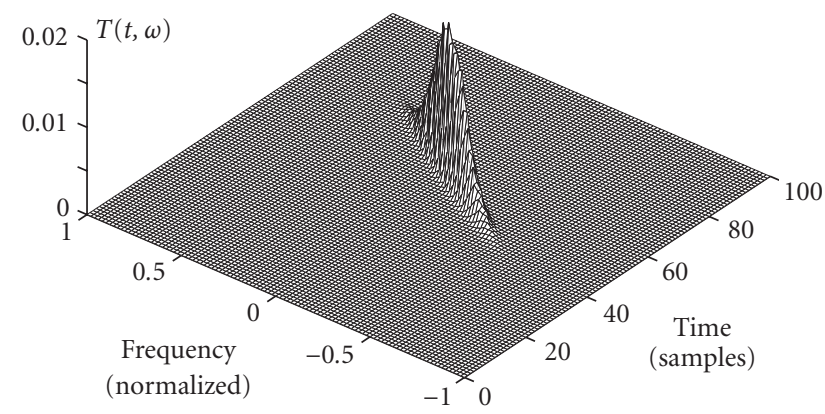

(b)

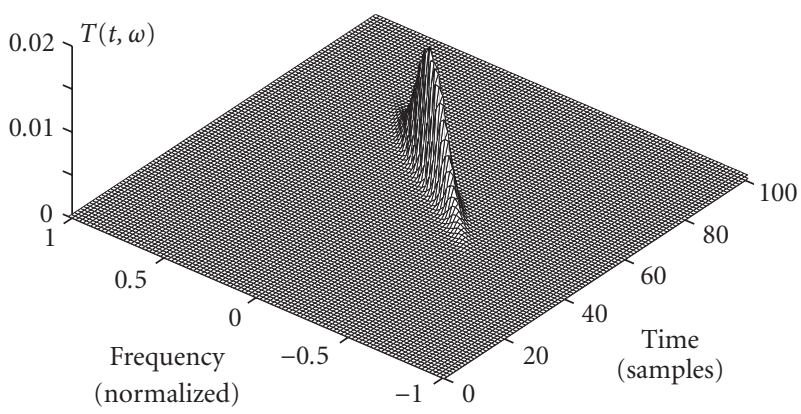

(c)

Figure 1: Reconstructed time-frequency atom based on (a) Rényi entropy $\alpha=1.9$; (b) Shannon entropy; and (c) Rényi entropy $\alpha=$ 1.1 .

from [1], sign of such interference does not fluctuate on lines parallel to the line connecting the center points of components, but interference oscillates with alternating signs in other directions. Based on this interference geometry, one can conclude that mainly such Radon-Wigner transforms are influenced by the interference, for which the integration paths are near parallel to the line connecting center points of components, whereas interference contributions to other directions are attenuated due to integration. Proper choice of angles then allows obtaining almost interference-free distribution.

The FrFT represents an inner product (correlation) of the analyzed signal with a linearly frequency-modulated prototype. Thus the performance of the FrFT-based approach 


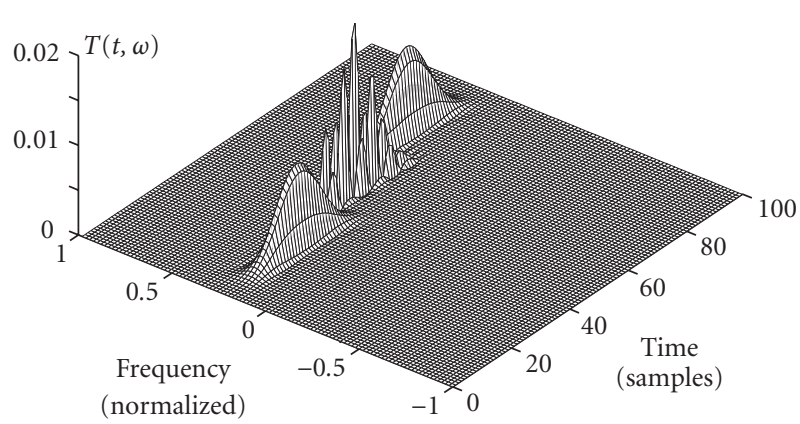

(a)

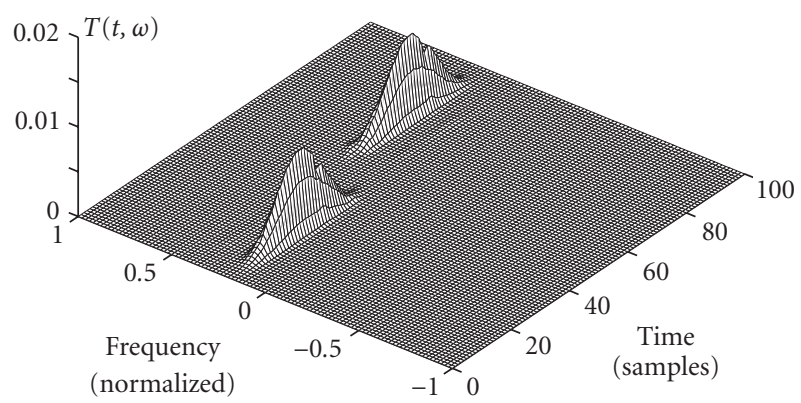

(b)

Figure 2: Time-frequency representations of two-component signal: (a) WD and (b) maximum entropy reconstruction from four angles.

in the case of nonlinear frequency modulation is inferior to the proposed method. As an example of a frequencymodulated signal with a higher order of nonlinearity, 201sample complex-valued signal with sinusoidal frequency modulation has been considered. The result of TFR reconstruction for nine angles $0^{\circ}, 20^{\circ}, \ldots, 160^{\circ}$ with $\alpha=1.1$ is given in Figure 3b. In this figure, complicated interference patterns along the main sinusoidal frequency modulation curve may be observed; also in comparison to Figure 2, it may be observed that interference terms have been redistributed from interference regions to autocomponent regions. Due to complex interference structure, no simple rule for choosing optimal set of angles may be found; uniform distribution of angles seems to be essential in this case. Compared to the signal with WD as in Figure 2, more angles must be used in order to reasonably characterize the signal structure in the time-frequency plane. However, as the number of projections increases, more interference patterns appear in the resulting distribution.

This method can be used also for CT originated data, as demonstrated in Figure 4, where Shepp-Logan phantom has been used to simulate measured projections.

\section{CONCLUSION}

In this paper, it has been explained that mainly limited-angle approach can contribute to the time-frequency analysis. The underlying concept of this novel method based on Rényi en-

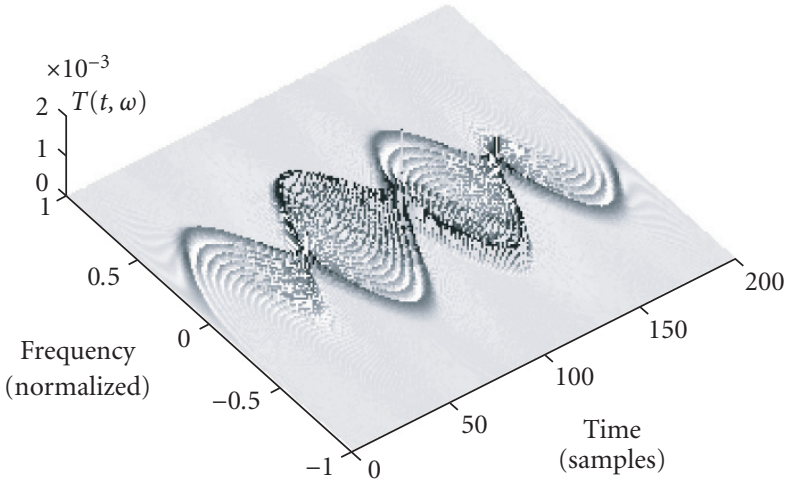

(a)

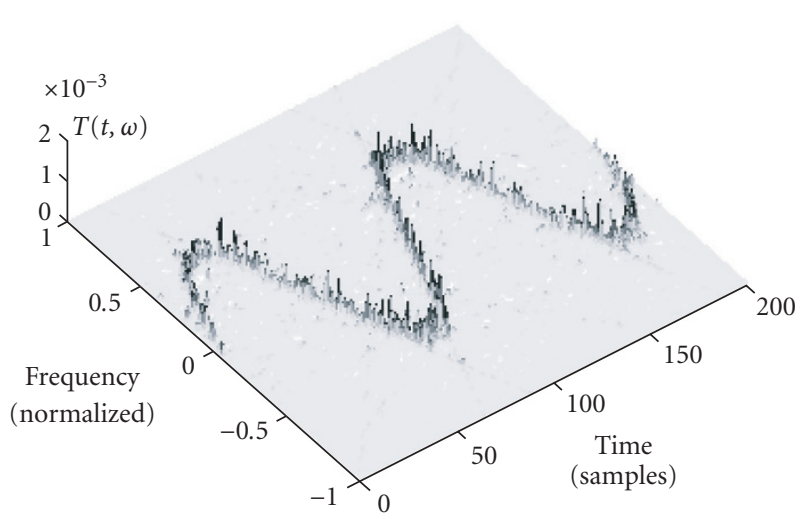

(b)

FIgURE 3: Time-frequency representations of a signal with sinusoidal frequency modulation: (a) WD and (b) the TFR reconstruction from 9 projections for $\alpha=1.1$.

tropy maximization has been described. This method can be used also for CT image reconstruction. Although this method is computationally very expensive, it can exhibit some advantages when compared to the conventional timefrequency methods. It has also been demonstrated that the proposed method can successfully suppress undesirable interference terms in the case of signals with simple timefrequency configurations without unacceptable degradation of time-frequency localization.

In the conclusion, it is worth noting the differences between the limited-angle approach in time-frequency reconstruction and tomography. Whereas a limited number of angles in tomography results from technical constraints, the angle limitation in time-frequency analysis has been imposed on purpose in order to increase the degree of freedom for the suppression of interference components. The aims of the construction procedures thus differ for these two fields of application. In tomography, the goal of image reconstruction is to achieve the highest level of detail possible, whereas in constructing the time-frequency distribution, the level of detail should not be tried to be arbitrarily small due to the uncertainty principle. Projections of images may not necessarily have a clear intuitive interpretation in signal theory; 


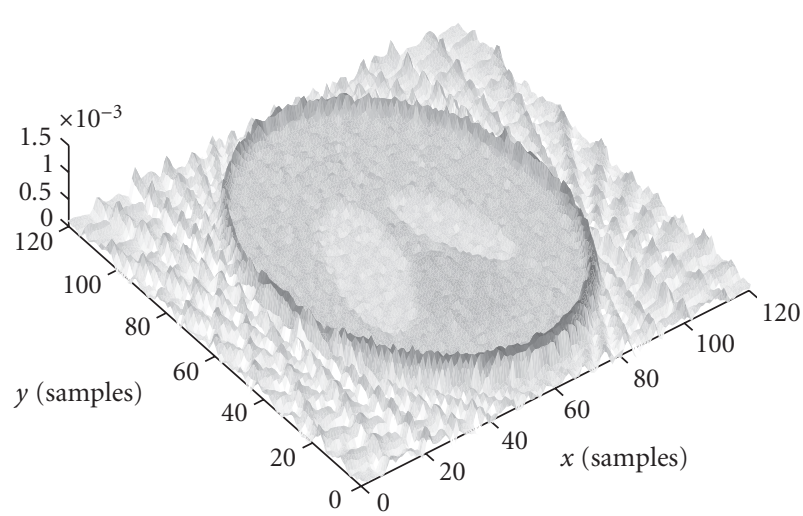

(a)

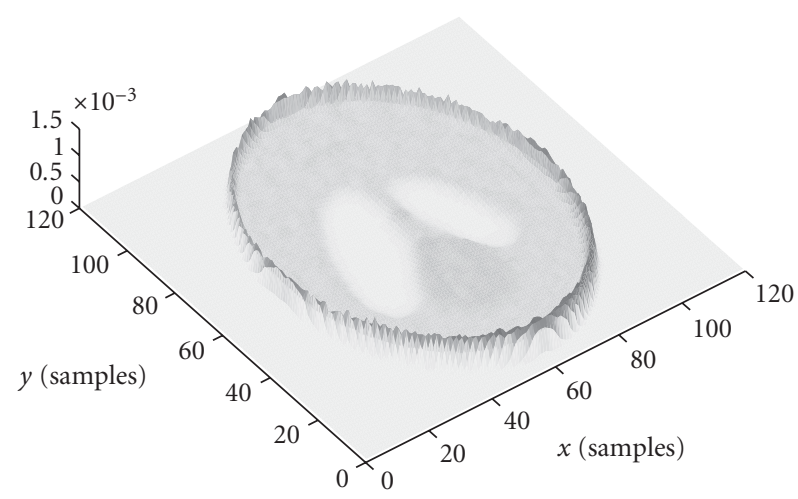

(b)

FIGURE 4: Shepp-Logan phantom reconstructed from 10 projections $(120 \times 120$ pixel $)$ by means of (a) filtered back projection and (b) Rényi entropy maximization, $\alpha=1.1$.

in contrast, time-frequency projections are related directly to the FrFT. This observation contributes to the theoretical value of the approaches presented.

\section{APPENDIX}

Iteration formula (22) can be treated as a functional mapping

$$
\lambda^{(n+1)}=\phi\left(\lambda^{(n)}\right) .
$$

Convergence of the iteration to the solution $(\lambda=\phi(\lambda)$, $\mathbf{e}(\boldsymbol{\lambda})=0$ ) can be ensured by contractivity of $\phi$. The mapping $\phi$ is said to be contractive if the inequality

$$
\left\|\phi\left(\lambda_{2}\right)-\phi\left(\lambda_{1}\right)\right\| \leq q\left\|\lambda_{2}-\lambda_{1}\right\|
$$

holds for a number $q<1$ and $\|\cdot\|$ denotes a properly defined norm. However, it is not easy to satisfy the contractivity condition "globally" for unbounded values of $\lambda$. In order to estimate the convergence parameter in (22), we consider "local" contractivity based on linearization with respect to $\lambda^{(n+1)}$ near $\lambda^{(n)}$. The linearized error term in (22) may be derived to obtain

$$
e_{i}\left(\lambda^{(n+1)}\right)=e_{i}\left(\lambda^{(n)}\right)+\mathcal{A}_{i}\left(\lambda^{(n+1)}-\lambda^{(n)}\right),
$$

where the linear operator $\mathcal{A}_{i}$ is defined as

$$
\mathcal{A}_{i} \mathbf{x}=\frac{1}{\alpha-1} \sum_{k=1}^{M} \mathcal{R}_{i}\left|\sum_{i=1}^{M} \mathcal{B}_{j} \lambda_{j}^{(n)}\right|^{(2-\alpha) /(\alpha-1)} \mathcal{B}_{k} x_{k} .
$$

Then (22) and (A.3) yield

$$
\phi\left(\lambda^{(n+1)}\right)-\phi\left(\lambda^{(n)}\right)=\left(\mathcal{I}-\mu_{n} \mathcal{A}\right)\left(\lambda^{(n+1)}-\lambda^{(n)}\right),
$$

where $\mathcal{A}=\left[\mathcal{A}_{1}, \ldots, \mathcal{A}_{M}\right]^{T}$ and $\mathcal{I}$ denotes the identity operator. From the definition of the operator norm induced by a function norm, it is clear that the local contractivity condition can be satisfied by imposing the constraint

$$
\left\|\mathcal{I}-\mu_{n} \mathcal{A}\right\|<1,
$$

where $\|\cdot\|$ is a corresponding operator norm.

In the next derivation, the $\infty$-norm will be used,

$$
\|\mathbf{x}(u)\|=\max _{i, u}\left\{\left|x_{i}(u)\right|\right\} .
$$

Taking into account the nonnegativity of the impulse response of $\mathcal{A}$, one can conclude that (A.6) can be satisfied by

$$
\left\|\mu_{n} \mathcal{A}\right\|<2, \quad \text { i.e., } \quad \mu_{n}<\frac{2}{\|\mathcal{A}\|},
$$

where the operator norm is

$$
\|\mathcal{A}\| \sup \|\mathcal{A} \mathbf{x}\|_{\|\mathbf{x}\|=1}=\max _{i}\left\{\sup \left\|\mathcal{A}_{i} \mathbf{x}\right\|\right\} .
$$

Operator $\mathcal{A}_{i}$ may be expressed in terms of a nonnegative kernel $h_{i, k}(u, v)$ as

$$
\left\{\mathcal{A}_{i} \mathbf{x}\right\}(u)=\sum_{k=1}^{M} \int h_{i, k}(u, v) x_{k}(v) d v .
$$

Therefore,

$$
\left|\left\{\mathcal{A}_{i} \mathbf{x}\right\}(u)\right| \leq \sum_{k=1}^{M} \int h_{i, k}(u, v)\left|x_{k}(v)\right| d v=\left\{\mathcal{A}_{i}|\mathbf{x}|\right\}(u)
$$

and $x_{k}(u)=1$ corresponds to the worst case of $\|\mathbf{x}\|=1$ with respect to supremum. Substituting $\mathbf{x}=[1, \ldots, 1]^{T}$ into (A.4) yields

$$
\mathcal{A}_{i}[1, \ldots, 1]^{T}=\frac{M}{(\alpha-1)}\left\{\mathcal{R}_{i}\left|\sum_{j=1}^{M} \mathcal{B}_{j} \lambda_{j}^{(n)}\right|^{(2-\alpha) /(\alpha-1)}\right\}(u) .
$$

This result, when combined with (A.8) and (A.9), finally results in the estimate (24). 


\section{ACKNOWLEDGMENT}

This work was supported by the Ministry of Education of the Slovak Republic under the Grant no. 1/0144/03.

\section{REFERENCES}

[1] F. Hlawatsch and G. F. Boudreaux Bartels, "Linear and qaudratic time-frequency signal representations," IEEE Signal Processing Magazine, vol. 9, pp. 21-67, April 1992.

[2] G. F. Boudreaux-Bartels and R. Murray, "Time-frequency signal representation for biomedical signals," in The Biomedical Engineering Handbook, J. D. Bronzino, Ed., pp. 866-885, CRC press, Boca Raton, Fla, USA, 1995.

[3] W. J. Williams, "Recent advantages in time-frequency representations: some theoretical foundations," in Time Frequency and Wavelets in Biomedical Signal Processing, M. Akay, Ed., pp. 3-43, IEEE Press, Piscataway, NJ, USA, 1998.

[4] L. Cohen, "Generalized phase-space distribution functions," J. Math. Phys., vol. 7, no. 5, pp. 781-786, 1966.

[5] L. Cohen and T. E. Posch, "Positive time-frequency distribution functions," IEEE Trans. Acoust. Speech Signal Processing, vol. ASSP-33, no. 1, pp. 31-38, 1985.

[6] P. Loughlin, J. W. Pitton, and L. Atlas, "Construction of positive time-frequency distributions," IEEE Trans. Signal Processing, vol. 42, no. 10, pp. 2697-2705, 1994.

[7] J. R. Fonollosa, "Positive time-frequency distributions based on joint marginal constraints," IEEE Trans. Signal Processing, vol. 44, no. 8, pp. 2086-2091, 1996.

[8] H. M. Ozaktas, N. Erkaya, and M. A. Kutay, "Effect of fractional Fourier transformation on time-frequency distributions belonging to the Cohen class," IEEE Signal Processing Letters, vol. 3, no. 2, pp. 40-41, 1996.

[9] H. M. Ozaktas, O. Arikan, M. A. Kutay, and G. Bozdagi, "Digital computation of the fractional Fourier transform," IEEE Trans. Signal Processing, vol. 44, no. 9, pp. 2141-2150, 1996.

[10] L. B. Almeida, "The fractional Fourier transform and timefrequency representations," IEEE Trans. Signal Processing, vol. 42, no. 11, pp. 3084-3091, 1994.

[11] D. Groutage, "A fast algorithm for computing minimum cross-entropy positive time-frequency distributions," IEEE Trans. Signal Processing, vol. 45, no. 8, pp. 1954-1970, 1997.

[12] S. I. Shah, P. Loughlin, L. F. Chaparro, and A. El-Jaroudi, "Informative priors for minimum cross-entropy positive timefrequency distributions," IEEE Signal Processing Letters, vol. 4, no. 6, pp. 176-177, 1997.

[13] G. Minerbo, "MENT: a maximum entropy algorithm for reconstructing a source from projection data," Computer Graphics and Image Processing, vol. 10, no. 1, pp. 48-68, 1979.

[14] A. Rényi, "On measures of entropy and information," in Proc. 4th Berkeley Symp. Math. Statist. Probab., vol. 1, pp. 547-561, University of California Press, Berkeley, Calif, USA, 1961.

[15] L. L. Campbell, "A coding theorem and Rényi's entropy," Information and Control, vol. 8, no. 4, pp. 423-429, 1965.

[16] R. G. Baraniuk, P. Flandrin, A. J. E. M. Janssen, and O. J. J. Michel, "Measuring time-frequency information content using the Rényi entropies," IEEE Transactions on Information Theory, vol. 47, no. 4, pp. 1391-1409, 2001.

[17] W. J. Williams, "Reduced interference distributions: biological applications and interpretations," Proceedings of the IEEE, vol. 84, no. 9, pp. 1264-1280, 1996.

[18] I. Kazantsev, R. Van de Walle, and I. Lemahieu, "Reconstruction from magnetic resonanse data based on ridge functions," in Proc. EURASIP International Conference BIOSIGNAL 2000, pp. 216-218, Brno, Czech Republic, June 2000.
Jozef Púčik was born in Bratislava, Slovakia, in 1972. He obtained the M.S. degree in 1996 and the Ph.D. degree in 2000 in electrical engineering from the Slovak University of Technology in Bratislava. Currently, he is an Assistant Professor at the Department of Radio and Electronics, Faculty of Electrical Engineering and Information Technology of the Slovak University of Technology in Bratislava. His main field of interest covers theory of time-frequency distributions and their application to biomedical signal processing.

Rami Oweis was born in Jordan in 1970. He received the B.S., M.S., and Ph.D. degrees in electronic engineering with the major biomedical instrumentation from the Slovak University of Technology. From 19941995, he worked as an Engineer in the Cardiovascular Department at the children university hospital with policlinic in Bratislava. From 1999, he is affiliated with the Jordan University of Science and Technology at the

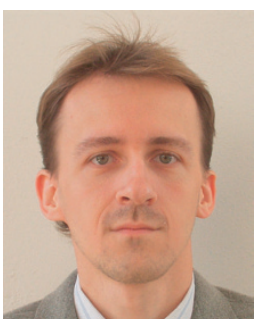
Biomedical Engineering Department. His research interests are biomedical electronics, and signal and image processing. 